\title{
Fabrication method of ultra-small gradient-index fiber probe
}

\author{
Chi Wang $\cdot$ Fang Zhang $\cdot$ Shu-Bo Bi $\cdot$ \\ Xue-Qin Xia $\cdot$ Ting-Ting Xu
}

Received: 17 June 2014/ Accepted: 11 September 2014/Published online: 14 October 2014

(c) Shanghai University and Springer-Verlag Berlin Heidelberg 2014

\begin{abstract}
Fabrication method and device of ultra-small gradient-index (GRIN) fiber probe were investigated in order to explore the development of ultra-small probes for optical coherence tomography (OCT) imaging. The beamexpanding effect of no-core fiber (NCF) and the focusing properties of the GRIN fiber lens were analyzed based on the model of GRIN fiber probe consisting of single-mode fiber (SMF), NCF and GRIN fiber lens. A stereo microscope based system was developed to fabricate the GRIN fiber probe. A fiber fusion splicer and an ultrasonic cleaver were used to weld and cut the fiber respectively. A confocal microscopy was used to measure the dimensions of probe components. The results show that the sizes of probe components developed are at the level of millimeter. Therefore, the proposed experimental system meets the fabrication requirements of an ultra-small self-focusing GRIN fiber probe. This shows that this fabrication device and method can be employed in the fabrication of ultrasmall self-focusing GRIN fiber probe and applied in the study of miniaturized optical probes and OCT systems.
\end{abstract}

Keywords Optical coherence tomography (OCT) . Gradient-index (GRIN) fiber probe · GRIN fiber lens · No-core fiber (NCF)

\section{Introduction}

Optical coherence tomography (OCT) has shown the wide application prospect in the fields such as biomedical and

C. Wang $(\bowtie) \cdot$ F. Zhang $\cdot$ S.-B. Bi $\cdot$ X.-Q. Xia $\cdot$ T.-T. Xu Department of Precision Mechanical Engineering, Shanghai University, Shanghai 200072, People's Republic of China e-mail: wangchi@shu.edu.cn surgical operations due to its advantages including rapid imaging speed, high resolutions, and non-contact detection, etc. [1] In an OCT system, an optical probe transmits and focuses a light beam (e.g., Gaussian beam) inside a sample for test, and then it collects the reflected or scattered light carrying information about the sample, and sends the information to the signal processing system. The optical probe is a key component of the OCT system, and its focusing performance plays an important role in determining the imaging quality of the OCT system. For example, the waist location, spot size and Rayleigh range of the Gaussian beam focused by the probe determine the penetration depth, lateral resolution and depth of OCT imaging field. However, most biological tissues are optically nontransparent, hence the penetration depth of OCT technology is limited, generally in the range of 1-3 $\mathrm{mm}$. The development of small or ultra-small optical probes has become an important growing branch of OCT technology with a view to making full use of OCT technology with high resolution, overcoming the limited penetration depth and avoiding possible injury to tissues.

In the process of studying the miniaturization of the optical probe and OCT systems, the gradient-index (GRIN) lens has been favored by researchers due to its good focusing performance, flat end face and easy integration with other components. For example, Xie et al. [2-4] designed a GRIN lens rod based probe for OCT endoscope system with fast dynamic focusing properties; Singh et al. [5] explored a miniature OCT probe with a scanning micro reflector; Aljasem et al. [6] developed a miniaturized fiber probe whose diameter was about $4 \mathrm{~mm}$; Meemon et al. [7] studied the method of designing an OCT endoscope system with dynamic focusing and high resolution; Min et al. [8] proposed the technology to design a fiber-based hand-held scanning probe. In addition, Jung et al. [9] presented a 


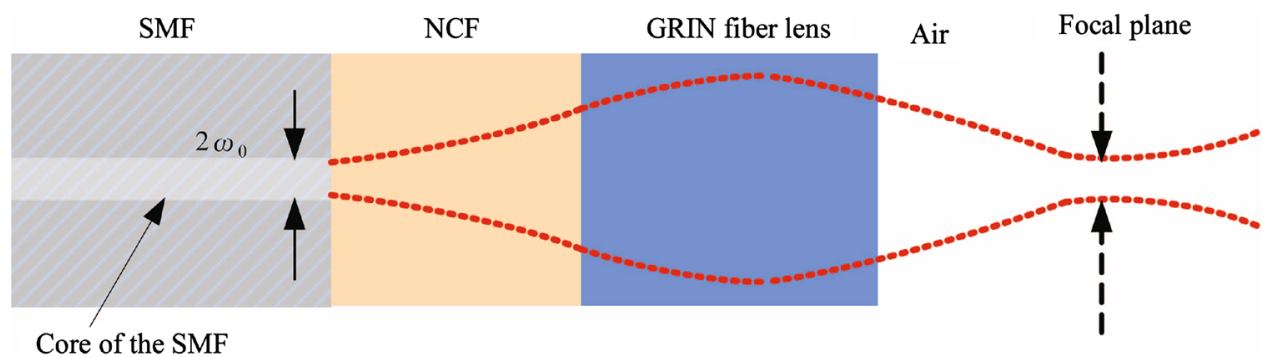

Fig. 1 Schematic diagram of GRIN fiber probe

numerical simulation method to design GRIN optical probes. However, the optical probes mentioned above are limited to be applied in the diagnosis of gastrointestinal tracts, arteries and other relatively large tissues or organs as their dimensions are all in a few millimeters. This method is unsuitable for the imaging detection in deep, narrow tissues or organs (such as cardiovascular system). For this reason, it is necessary to develop ultra-small optical probes with good biological mechanical compatibility.

The GRIN fiber probe is defined as an all-fiber-type ultra-small optical probe for miniaturization of OCT system, typically consisting of a single-mode fiber (SMF), a no-core fiber (NCF), and a GRIN fiber lens (see Fig. 1). In 2002, Swanson et al. applied a USP of GRIN fiber lens-based ultra-small optical probe [10]. Reed and Jafri et al. [11-13] developed the OCT online imaging system based on the patent. Mao et al. [14, 15] studied the fabrication and performance testing methods of GRIN fiber probes, making substantial progress in the study of GRIN optical fiber probes. However, there are no reports about detailed theoretical analysis of the probe. In 2011, our research group began to study the design method of ultra-small GRIN fiber probes from analytical and numerical simulation and other aspects [16-21], while no making research into fabrication. In this paper, on the basis of existing research, we explore the fabrication of the GRIN fiber probe and design the fiber cutting and fusing system based on the stereo microscope, providing a technical foundation for the study of ultra-small OCT systems based on the GRIN optical fiber probe.

\section{Model of the GRIN fiber probe}

GRIN optical fiber probe, as a kind of ultra-small optical probe, can be adapted for the study of the miniaturized OCT systems used in vivo and online detection of biological tissues or organs, which are deep and narrow (such as the cardiovascular system). Figure 1 represents a typical model of a GRIN optical fiber probe, consisting of an
SMF, an NCF and a GRIN fiber lens. The SMF, connected with the detection arm of the OCT system, guides the light beam into the NCF. As a kind of special fiber with the same index of refraction, the NCF is able to overcome the limited mode field diameter of the SMF by an expanding beam, thus improving the focusing performance of the probe. However, the NCF may also reduce the lateral resolution of OCT imaging by increasing the size of the focus spot. Therefore, the length of the NCF should be chosen appropriately. If the NCF is too long, some light energy will overflow the probe side, which may finally reduce the beam coupling efficiency and eventually lower the sensitivity of the OCT system. In addition, a very short NCF may result in the failure of the expanding beam and thus the probe's performance cannot be improved.

As the most critical part of the fiber probe, GRIN fiber lens performs a self-focusing function because of the continuous change of refractive index. It is convenient to fuse the lens with other optical elements with plane end surfaces due to its flat surface, thus improving the mechanical strength and stability of the probe. There is no doubt that the length of the GRIN fiber lens should be moderate. If it goes close to $1 / 4$ pitch (or the integer times), the lens will have a strong focusing performance and a short focal length. If the lens's length is close to $1 / 2$ pitch (or the integer times), a longer focal length will be obtained, and a bigger spot size. In the study of the imaging performance of an OCT system, a greater focal length is desired in order to obtain a larger detection depth, and a small focusing spot size is required to get a higher lateral resolution. Therefore, a tradeoff is needed in determining the length of GRIN fiber lens. An ideal design for a probe can be obtained by using a coreless fiber to improve the focusing performance based on its beam-expanding function. Besides, to minimize the influence of the complicated reflection of input beam by different optical interfaces on OCT imaging signals for detection, and to obtain a higher signal-to-noise ratio, the refractive indices of the NCF and the core of GRIN fiber at the axis should match that of the SMF at the core. 


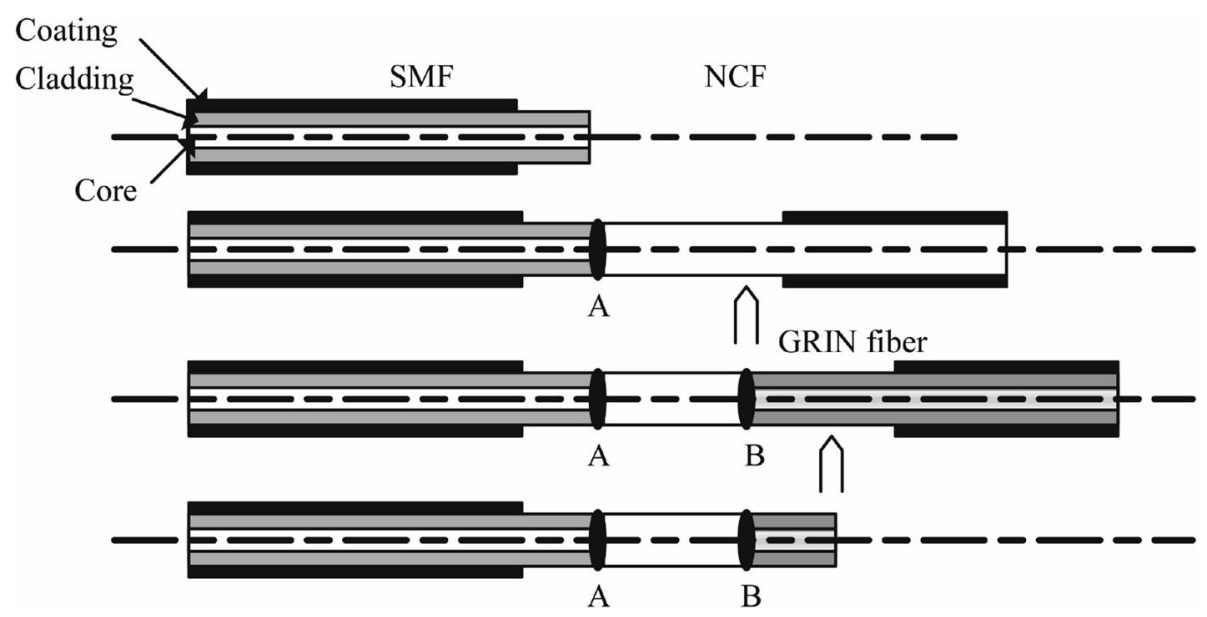

Fig. 2 Process of fabricating a GRIN optical fiber probe

\section{Fabrication method and device of the GRIN fiber probe}

In general, the lengths of the NCF and the GRIN fiber lens in the GRIN optical fiber probe are in the millimeter or submillimeter range, a very short size. Therefore, high precision cutting and welding of the optical fiber lengths are required in the fabrication of the optical fiber probe. In this paper, the fabrication process of the GRIN fiber is shown in Fig. 2. The main steps are as follows: (i) Fuse the NCF to the single-mode optical fiber; (ii) Cut the NCF to a certain length as fiber spacer by taking the fusion point $\mathrm{A}$ as the origin between SMF and NCF; (iii) Fuse the GRIN fiber lens to the fiber spacer; (iv) Cut GRIN fiber to precalculated length as the focusing lens by taking the welding joint $B$ as the origin between the fiber spacer and the GRIN fiber lens.

\subsection{Welding process of optical fiber components}

Different fiber components of the GRIN fiber probe are welded using the Fusion Splicer S117A made by Japanese Furukawa Electric Industrial Co. (see Fig. 3). As shown in Fig. 3a, there are two optical fiber slots, one for optical fiber 1 (such as SMF) and the other for optical fiber 2 (such as NCF). Meanwhile, the end planes of the two optical fibers should be aligned as accurately as possible and located in the middle between the two welding interfaces. The fusion splicer will be closed and then the power will be switched on after the optical fibers are put into their places. As shown in Fig. 3b, in order to reach a better welding performance, the locations of the two optical fibers will be adjusted by the fusion splicer after it is put into operation. Figure $3 \mathrm{c}$ shows the parameters about the welding quality. The energy loss rate of the welding joints (i.e., welding spot), which is only $0.02 \mathrm{~dB}$, proves that the good welding performance is achieved. As the fusion splicer S117A is of a high automatic level, once two different kinds of fiber probes are put into the slots, the adjustment to precise positions, welding process, welding performance evaluation and other parameters, will be completed automatically. Thus, the fusion splicer can accomplish the fusion of different fibers at high quality. However, the welding spot joining the different fibers is not obvious by using this high quality welding machine, which makes it difficult to cut the optical fibers to the pre-calculated length with high precision.

\subsection{Cutting process of optical fiber components}

Due to the high welding quality of the fusion splicer S117A, the influence of reflection caused by the beam going through the welding spot to the detection sensitivity and signal-to-noise ratio of the OCT system is reduced structurally. The small welding spot left makes it hard to cut the probe components to the pre-calculated length accurately. In this paper, therefore, a high-precision fiber cutting system is designed based on the stereo microscope XTL-2400 (Zhoushan Precise Optical Instrument Co., Ltd in Shanghai, China) and ultrasonic fiber cleaver FKII-4 (PK TECHNOLOGY, USA). As shown in Fig. 4, the fiber cleaver FKII-4 is placed on the workbench of the microscope XTL-2400. The optical fiber cutting process is as follows. Firstly, the optical fiber for cutting (the structure is spliced using two kinds of fibers, such as SMF and NCF) is placed in the slot of the ultrasonic cutting machine, and the position of the welding spot is aligned with the knife of the ultrasonic cutting machine. Secondly, the magnification and view field of the microscope should be adjusted to observe the welding spot and the blade of ultrasonic cutting machine from the eyepiece lens with scale lines. Thirdly, the probe is moved to the right position so that the blade 




(a) Inner structure

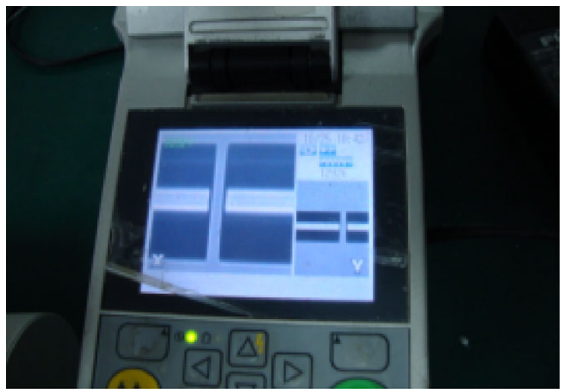

(b) External display (before welding)

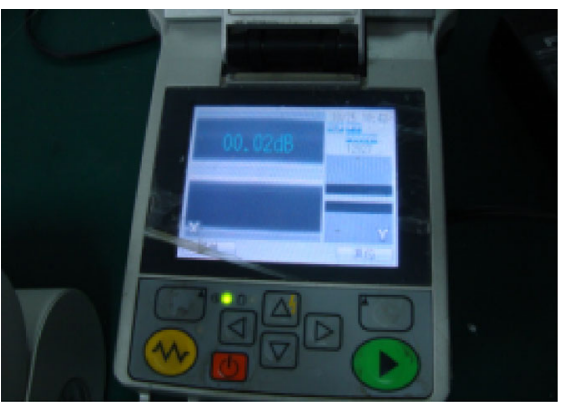

(c) External display (after welding)

Fig. 3 Fiber fusion splicer

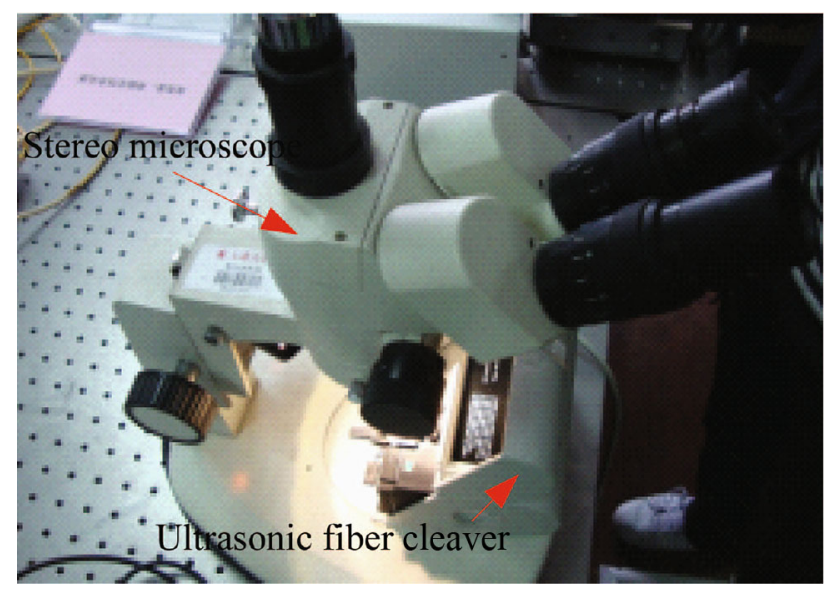

Fig. 4 Fiber cutting system

remains at the pre-calculated length from the welding spot. Finally, the ultrasonic cutting machine is switched on, and the fiber is cut at a certain length by an ultrasound pulse with high energy intensity. The energy pulse given out by the blade of the ultrasonic cutting machine has a small bandwidth (about a few micrometers), hence high-precision fiber cutting can be achieved.

\section{Measurement of the size of GRIN fiber probe}

Although the fiber cleaver FK II-4 and the fusion splicer S117A are of high cutting accuracy and welding precision respectively, the performance of the probe will be affected by the error between the pre-calculated length and the actual cutting length resulting from the position of the placed probe relative to the tilting angle of blade due to the ultra-small size of the probe. Therefore, the dimensions of a completed GRIN optical fiber probe need to be measured accurately.

The component size of the probe is examined with the laser scanning confocal microscope OLS4000 (Olympus

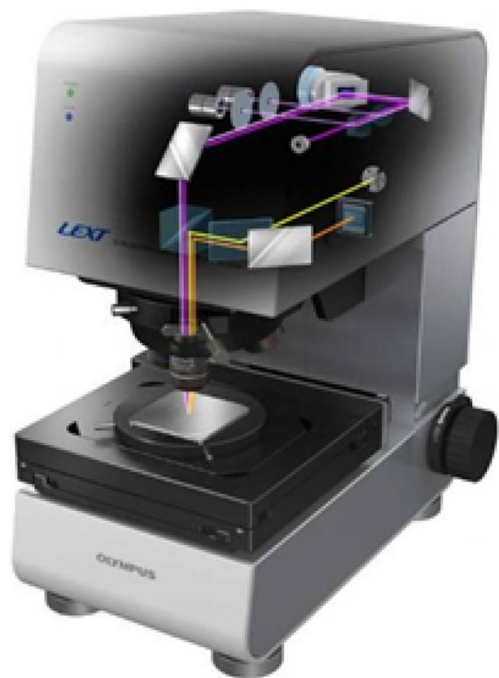

Fig. 5 Confocal microscopy OLS4000

Corporation), as shown in Fig. 5. Unlike the traditional focusing method by varying the height of object table, this kind of confocal microscope is focused through moving the objective lens up and down, which guarantees the stability of the lens body to the largest degree. Thus, superficial microscopic images with the horizontal resolution up to $0.12 \mu \mathrm{m}$ can be obtained. At the same time, the colorful image information collected with CCD is used to integrate into true color image forms of high resolution. Geometrical parameters in the submicron range including line width, area, volume, and steps can be measured and shown in the form of report.

Figure 6 shows the image of a sample GRIN optical fiber probe under the confocal microscope, which is fabricated with the cutting and welding system designed in this paper. In addition, the welding spots are magnified in particular. In Fig. 6, the position of the welding spots can be seen clearly under OLS4000, which can be used to measure the lengths of different optical fiber elements. Figure 7 shows the measuring window panel in computer. 


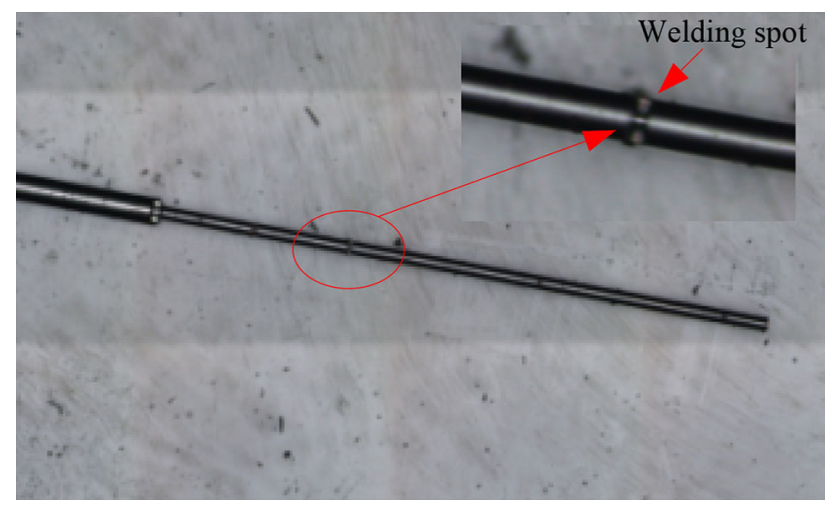

Fig. 6 GRIN fiber probe with welding spots

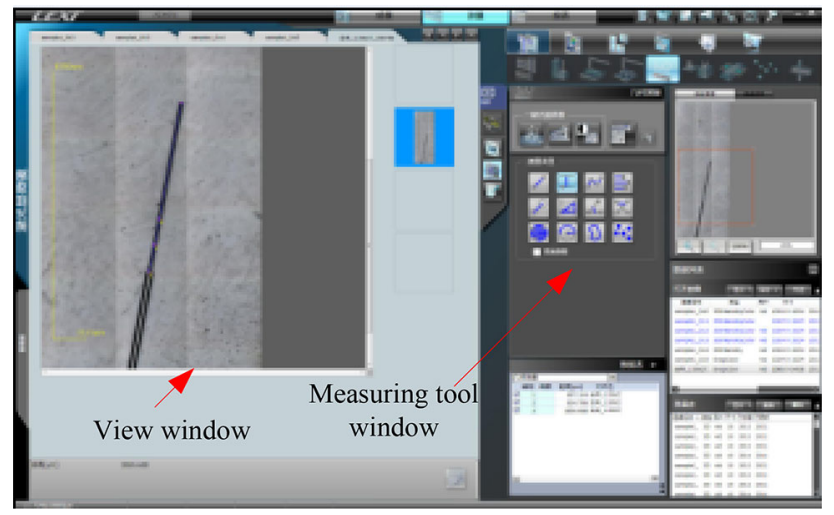

Fig. 7 Measurement window of dimensions of GRIN fiber probe

According to the measurement results, the distance from the probe without the coating layer to the first welding spot is $1.02 \mathrm{~mm}$ for an SMF, $2.37 \mathrm{~mm}$ for an NCF, and $2.26 \mathrm{~mm}$ for GRIN fiber lens. The size of the welding spots are very small, close to a few micrometers.

\section{Conclusions}

The study of ultra-small probes is an important topic for the miniaturization of OCT systems. As an all-fiber-type ultrasmall optical probe, the GRIN fiber probe is becoming increasingly promising for detection of optical imaging of the small lumen, narrow spaces in the deep tissues and organs (such as the cardiovascular system). Based on the model of the GRIN fiber probe presented in this paper, a set of experimental fabrication systems were designed, in which the experimental system based on stereo microscope and ultrasonic fiber cleaver was used to cut the fiber length with high-precision and the fusion splicer was used to weld different components together. In addition, the scheme to measure the size of probe based on the confocal microscope was also described. The designed experimental system can be used for the experimental study of the fabrication of GRIN fiber probes, providing the experimental foundation for the optimal design and the miniaturization of the OCT system.

Ackowlegments This project was supported by the National Natural Science Foundation of China (Grant No. 41104065), the Dawn Planning Foundation of Shanghai Municipal Education Commission (Grant No. 12CG047), and by the Scientific Research Innovation Project of Shanghai Municipal Education Commission (Grant No. 13YZ022).

\section{References}

1. Huang D, Swanson EA, Lin CP et al (1991) Optical coherence tomography. Science 254(5035):1178-1181

2. Xie T, Mukai D, Guo S et al (2005) Fiber-optic-bundle-based optical coherence tomography. Optics Lett 30:1803-1805

3. Xie T, Guo S, Chen Z et al (2006) GRIN lens rod based probe for endoscopic spectral domain optical coherence tomography with fast dynamic focus tracking. Optics Express 14:3238-3246

4. Sun J, Guo S, Wu L et al (2010) 3D in vivo optical coherence tomography based on a low-voltage, large-scan-range 2D MEMS mirror. Optics Express 18:12065-12075

5. Singh J, Teo JHS, Xu Y et al (2008) A two axes scanning SOI MEMS micromirror for endoscopic bioimaging. J Micromech Microeng 18:025001

6. Aljasem K, Werber A, Seifert A et al (2008) Fiber optic tunable probe for endoscopic optical coherence tomography. J Optics A 10:044012

7. Meemon P, Lee KS, Murali S et al (2008) Optical design of a dynamic focus catheter for high-resolution endoscopic optical coherence tomography. Appl Optics 47:2452-2457

8. Min EJ, Na J, Ryu SY et al (2009) Single-body lensed-fiber scanning probe actuated by magnetic force for optical imaging. Optics Lett 34:1897-1899

9. Jung W, Benalcazar W, Sharma U et al (2010) Numerical analysis of gradient index lens-based optical coherence tomography imaging probes. J Biomed Optics 15:066027

10. Swanson E, Petersen CL, McNamara E et al (2002) Ultra-small optical probes, imaging optics, and methods for using same. US Patent 6445939 B1, 3 Sept 2002

11. Reed WA, Yan MF, Schnitzer MJ (2002) Gradient-index fiberoptic microprobes for minimally invasive low-coherence interferometry. Optics Lett 27:1794-1796

12. Jafri MS, Schmitt JM, Farhang S et al (2005) Optical coherence tomography in the diagnosis and treatment of neurological disorders. J Biomed Optics 10:051603

13. Li H, Standish BA, Mariampillai A et al (2006) Feasibility of interstitial doppler optical coherence tomography for in vivo detection of microvascular changes during photodynamic therapy. Lasers Surg Med 38:754-761

14. Mao YX, Chang S, Sherif S et al (2007) Graded-index fiber lens proposed for ultrasmall probes used in biomedical imaging. Appl Optics 46:5887-5894

15. Mao YX, Chang S, Flueraru C (2010) Fiber lenses for ultra-small probes used in optical coherent tomography. J Biomed Sci Eng 3:27-34

16. Wang C, Mao YX, Fang C et al (2011) Analytical method for designing gradient-index fiber probes. Opt Eng 50:094202 
17. Wang C, Bi SB, Xia XQ et al (2014) Further analysis of focusing performance of an ultra-small gradient-index fiber probe. Opt Eng 53:013106

18. Wang C, Mao YX, Tang Z et al (2011) Numerical simulation of a gradient-index fibre probe and its properties of light propagation. Chin Phys B 20:114218

19. Wang C, Mao YX, Tang Z et al (2011) Numerical analysis of GRIN lens based miniature probes for optical coherence tomography. Opt Preci Eng 19:2300-2307
20. Wang C, Bi SB, Xia XQ et al (2013) Field-tracing based numerical simulation technique for the investigation of ultrasmall self-focusing optical fiber probe. Acta Phys 62:024217

21. Wang C, Bi SB, Wang L et al (2013) Field-tracing modeling of the ultra-small gradient-index fiber probe. OptiK Int J Light Electron Opt 124:6437-6443 